\title{
Fermi GBM Observations of Terrestrial Gamma-ray Flashes
}

Colleen A. Wilson-Hodge (NASA/MSFC), M.S. Briggs, V. Connaughton (UAH), G.J. Fishman (NASA/MSFC), P.N. Bhat, W.S. Paciesas, R. Preece (UAH), R.M. Kippen (LANL), A. von Kienlin (MPE), J. R. Dwyer (FIT), D.M. Smith (UCSC), R. Holzworth (Washington)
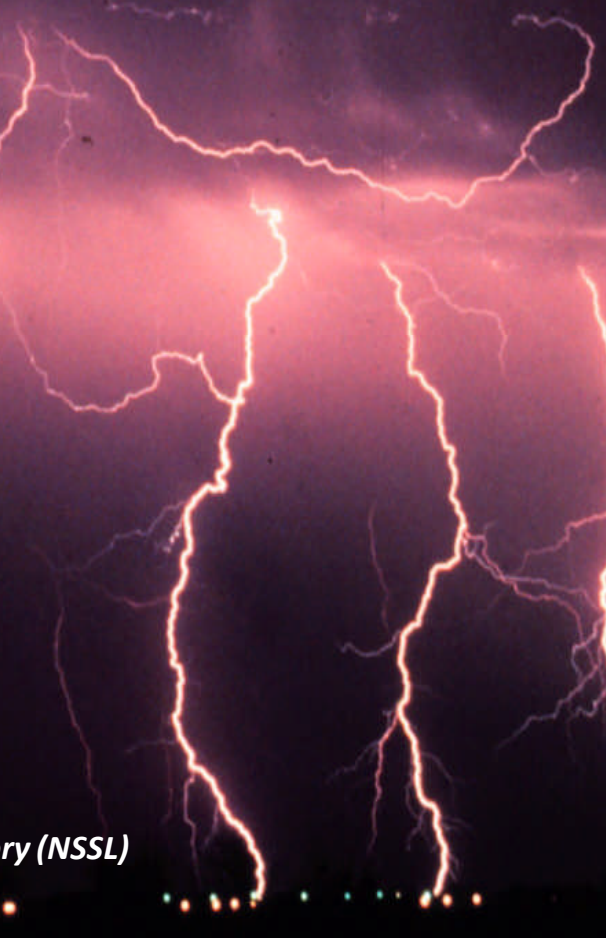


\section{Fermi Gamma-ray Burst Monitor}

-Fermi

- Launched June 11, 2008

- $565 \mathrm{~km}$ orbit

- 26.5 deg inclination

$\cdot \mathrm{GBM}$

-12 Nal detectors

- 8keV - $1 \mathrm{MeV}$

- 2 BGO detectors -150 keV - $40 \mathrm{MeV}$

- Triggers

- BGO triggers for TGFs

- Implemented Nov 2009

- 87 TGF triggers as Aug 27

- TGF trigger every $\sim 4$ days

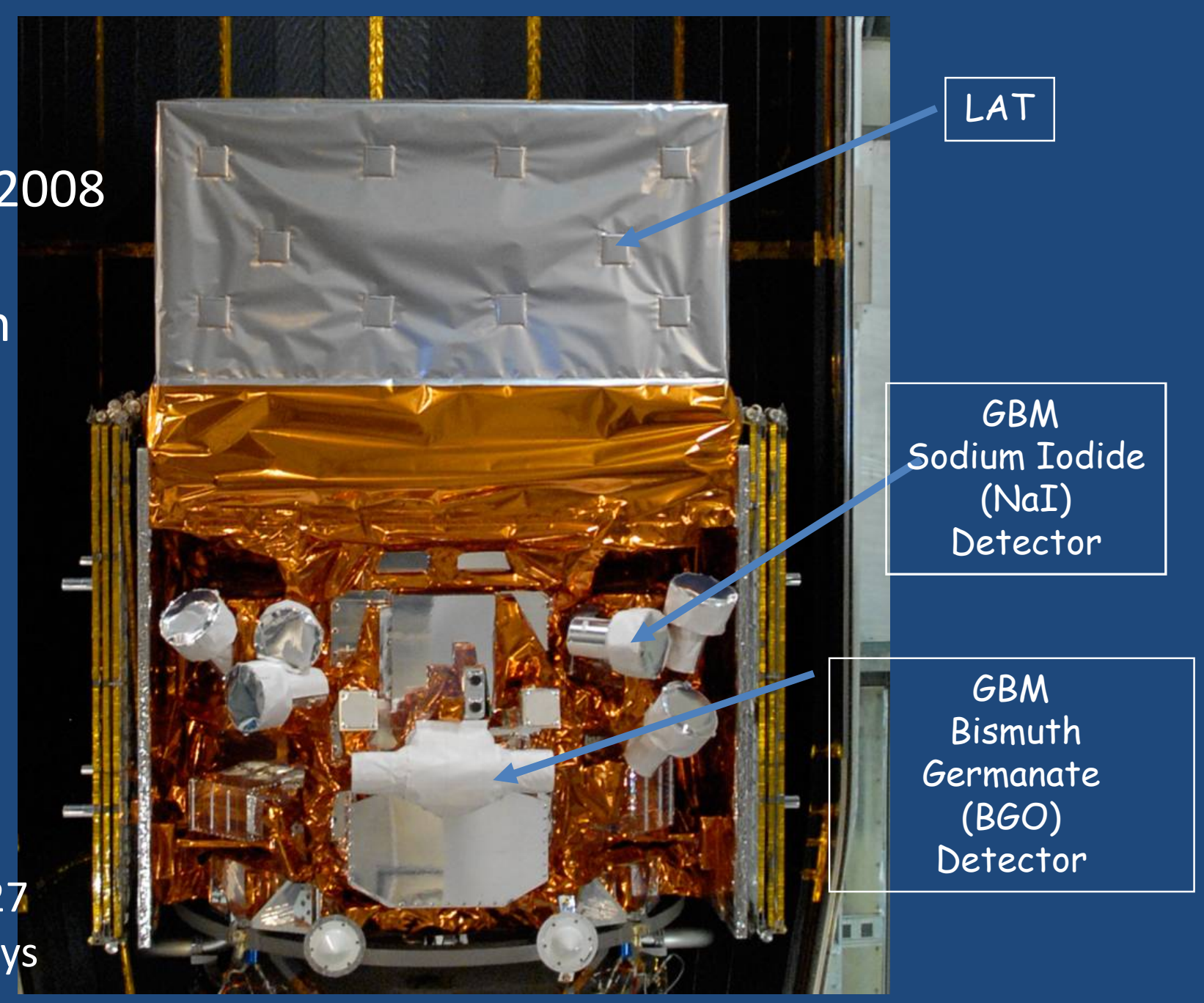




\section{Lightning \& GBM TGF Maps}

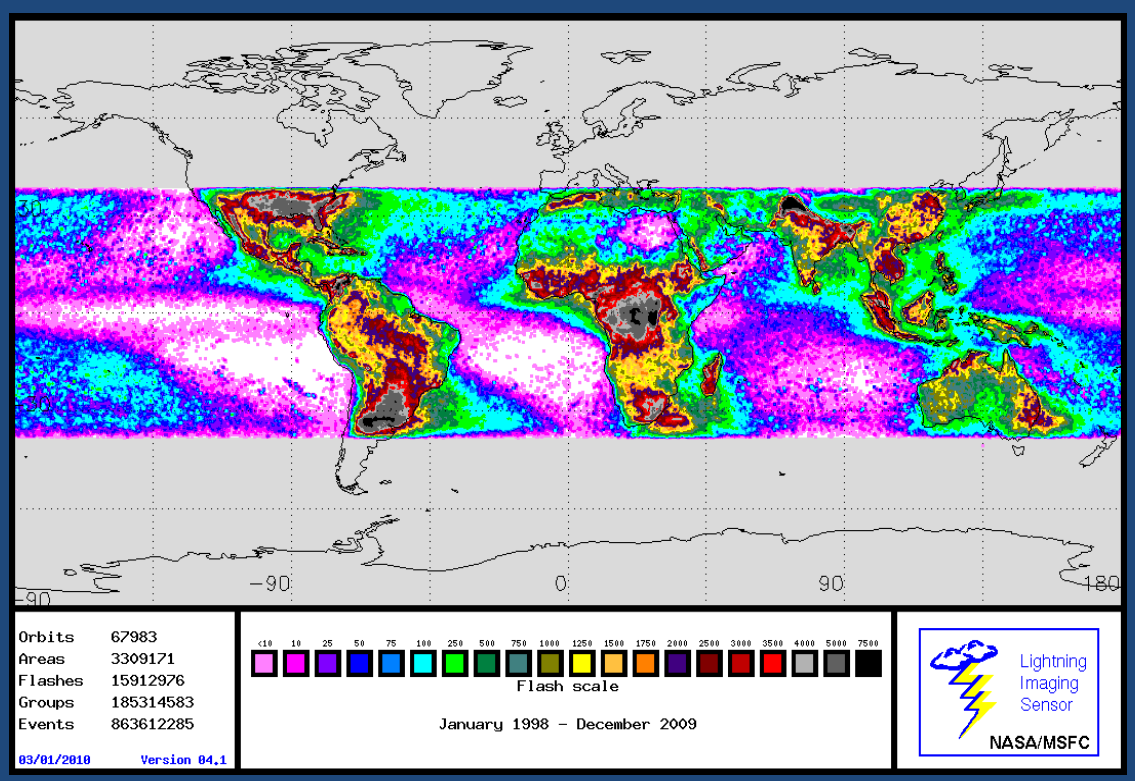

- Which comes first, lightning or TGF?

- Does one cause the other?

- Or do they have a common cause?

- Do they have a consistent time order?

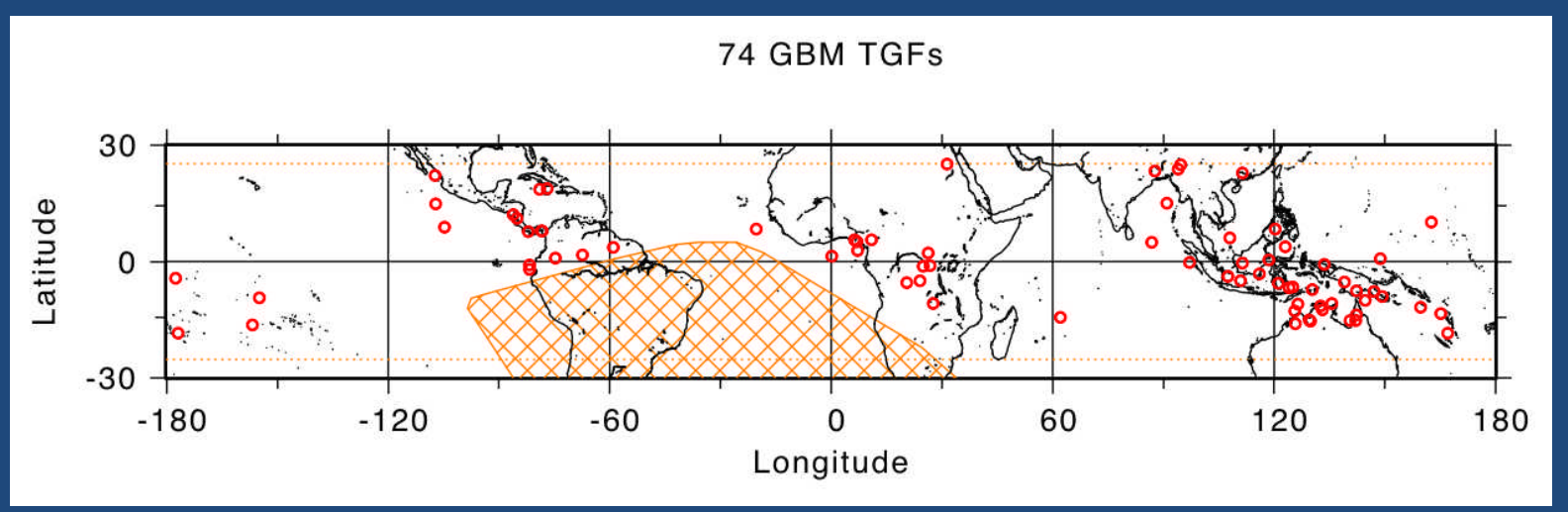

"Associations between Fermi GBM Terrestrial Gamma-ray Flashes and sferics from the WWLLN", V. Connaughton, et al, JGR in press. 


\section{WWLLN}

- World-Wide Lightning Location Network.

- $10 \%$ of lightning worldwide, $30 \%$ lightning with peak current $>30 \mathrm{kA}$.

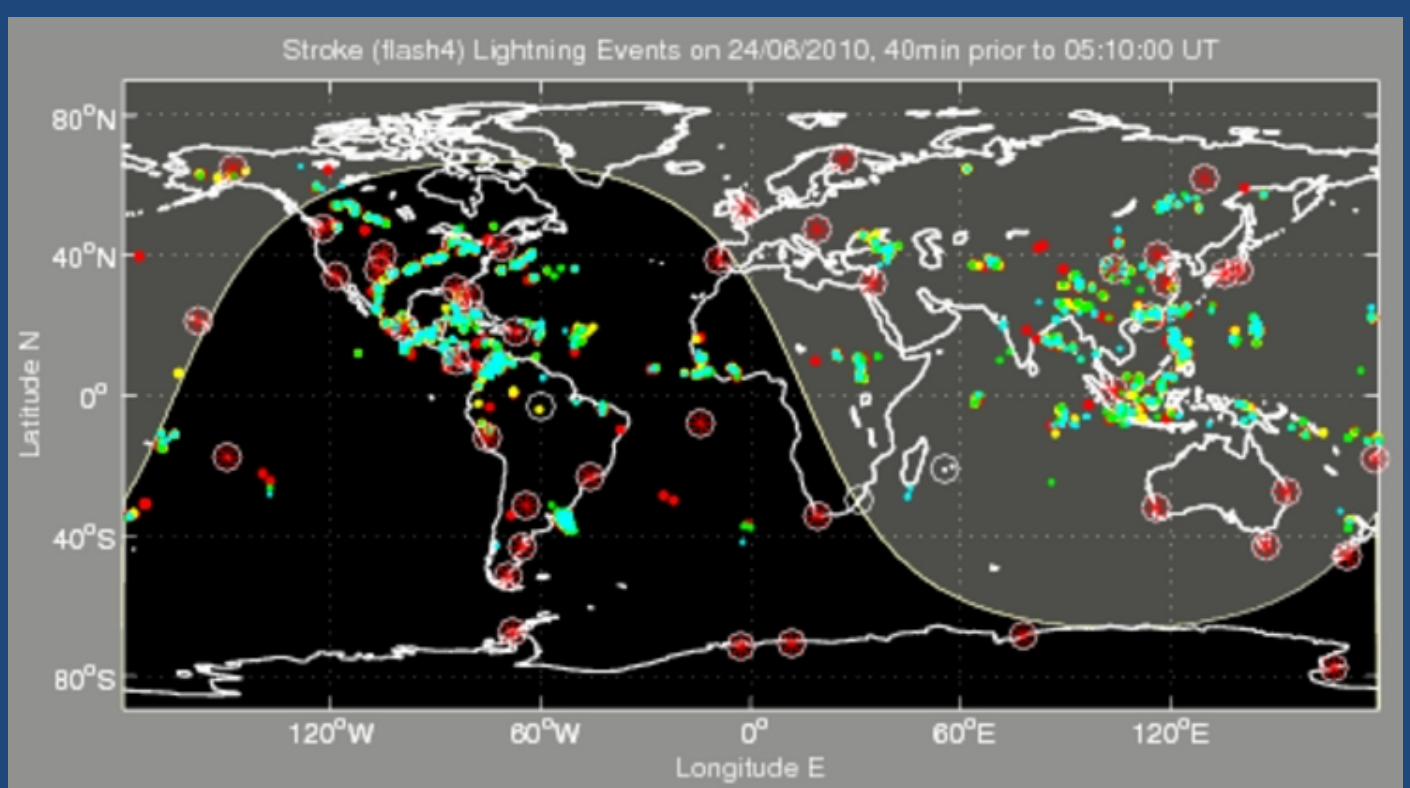

- Timing accuracy (with 5+ stations) $30 \mu$ s, geolocation $10 \mathrm{~km}$.

- No information on lightning type, discharge size, shape.

- http://wwlln.net headed by Bob Holzworth. 


\section{Detection distance}

- In first 50 GBM TGFs (July 2008-March 2010)

- $30 \%$ of GBM gamma-ray TGFs have a match with individual sferic within $5 \mathrm{~ms}$ of TGF peak and $1000 \mathrm{~km}$ of sub-spacecraft position.

- Blind searches reveal the false positive rate is very small (1-7 per 1000).

- All 15 matched sferics are within $300 \mathrm{~km}$ of sub-spacecraft point.
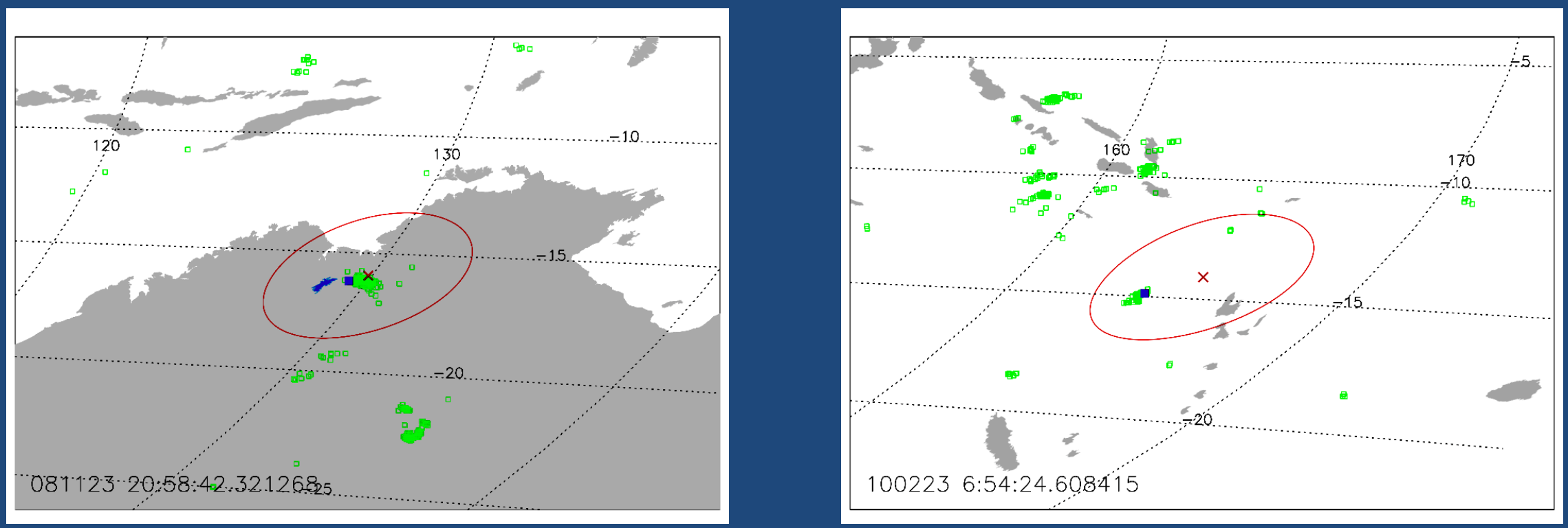

- Red - Fermi sub-spacecraft location with a $300 \mathrm{~km}$ radius

- Green - WWLLN lightning strokes within 10 minute of the GBM trigger time

- Blue Square - Exact match - sferic within $5 \mathrm{~ms}$ and 300 km 


\section{TGF-lightning are Simultaneous!}
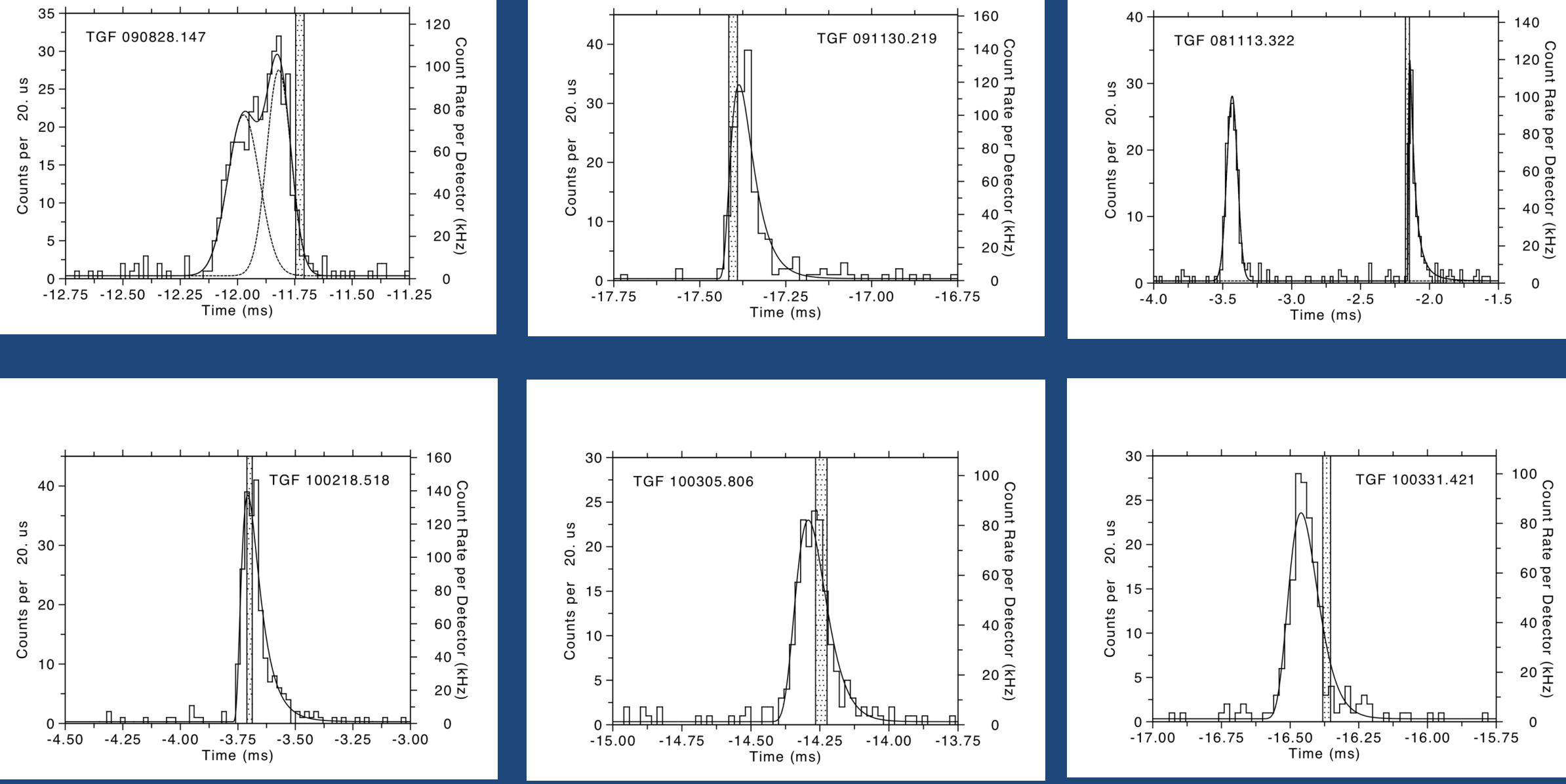

- GBM light curves corrected for light travel time and clock drift (histogram) -WWLLN stroke time and uncertainty band (dotted vertical bar) 


\section{How simultaneous?}

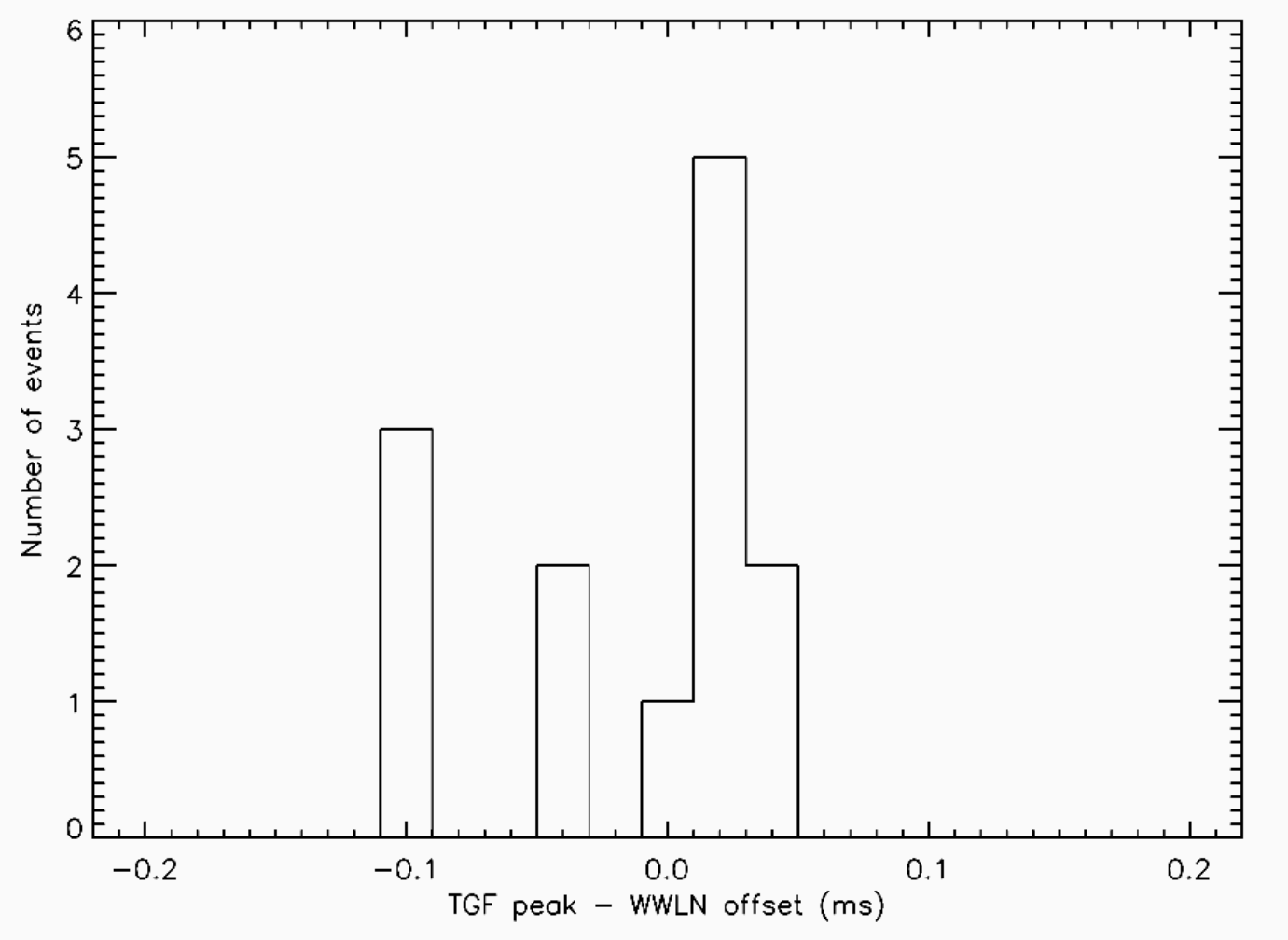

- Peak times of simultaneous TGFs and sferics agree to within $\sim 40 \mu \mathrm{s}$.

- No indication of preferred order.

- Two exceptions (not shown)

- Within $5 \mathrm{~ms}$ and $300 \mathrm{~km}$, but occur ms before or after the TGF peak.

- Likely explanation: TGF and sferic are associated with the same storm but not with each other. 


\section{Storms ... almost always!}

- In absence of sferic matches, (almost) always have storms within $300 \mathrm{~km}$ of sub-spacecraft position.

- Suggests 30 deg opening angle (including scattering) for detection from gamma-ray TGFs.
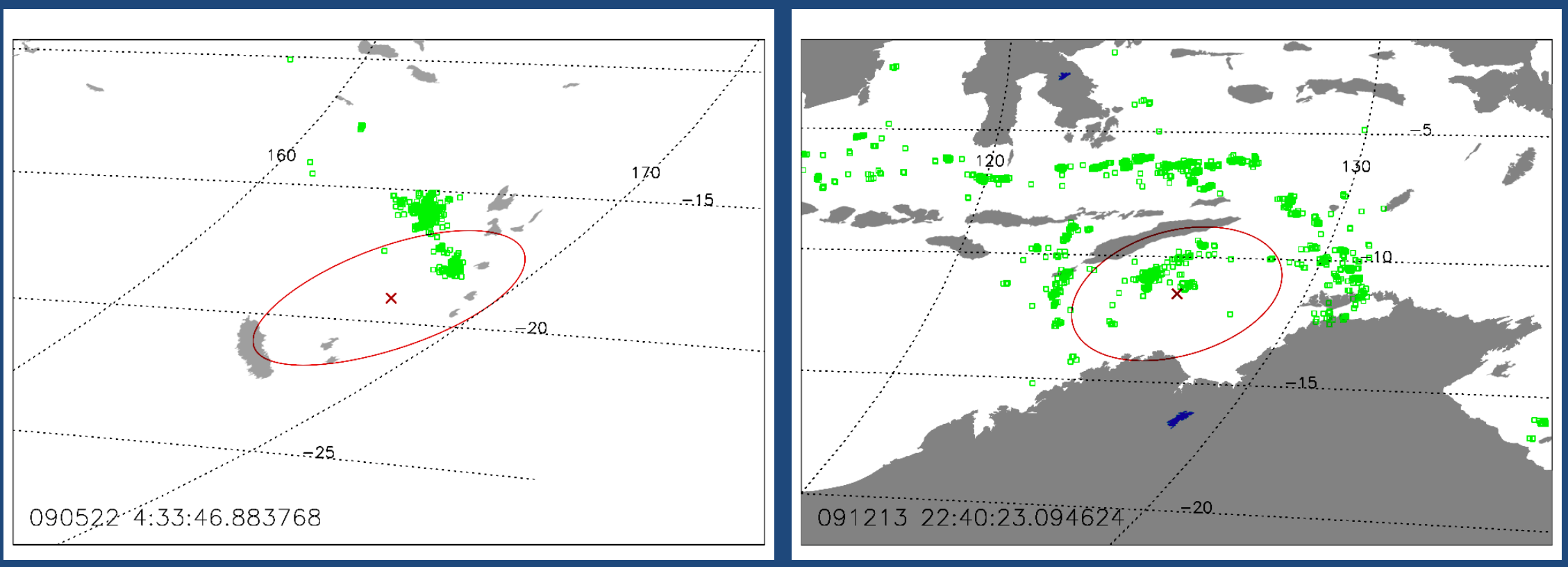


\section{No coincidence - no storm}

Sub-spacecraft

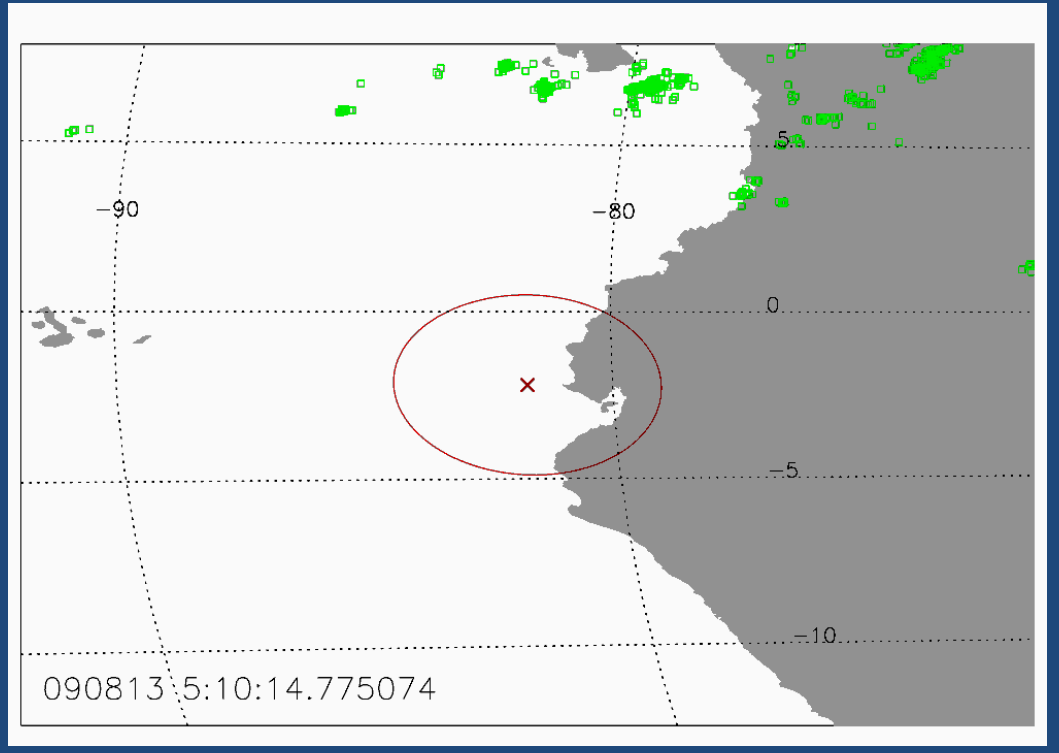

Magnetic footprint

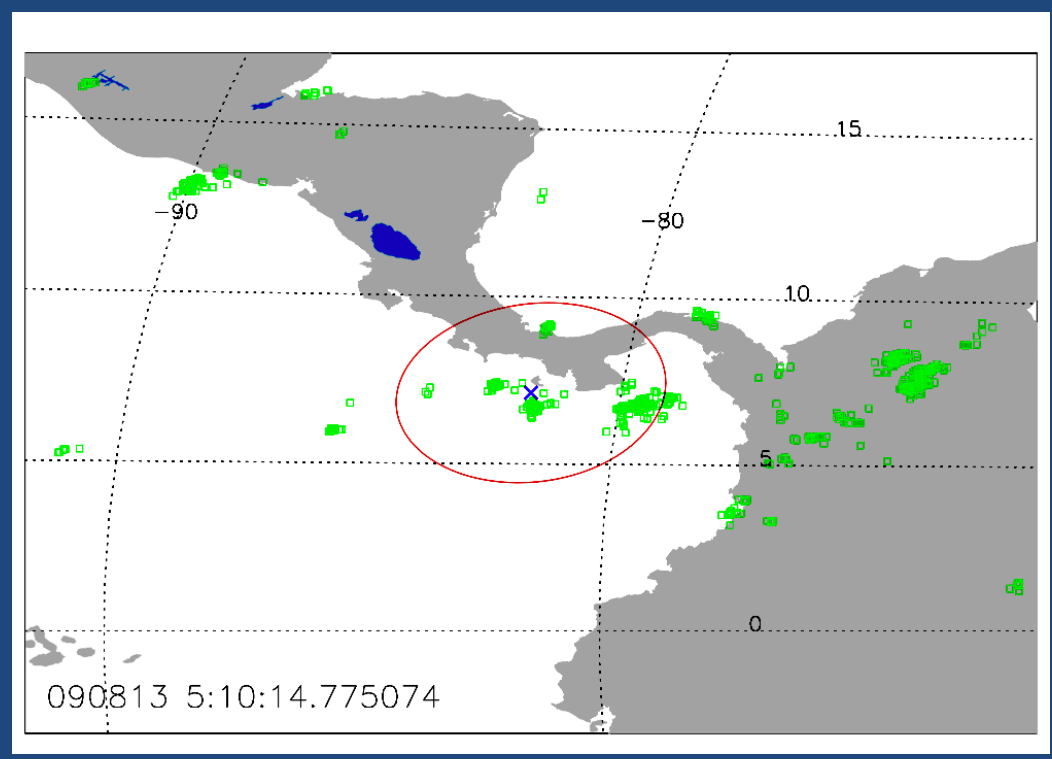

- In 3 of 4 cases with no storm under spacecraft.... storm activity at one of the geomagnetic footprints. All 4 cases look like electron TGFs.

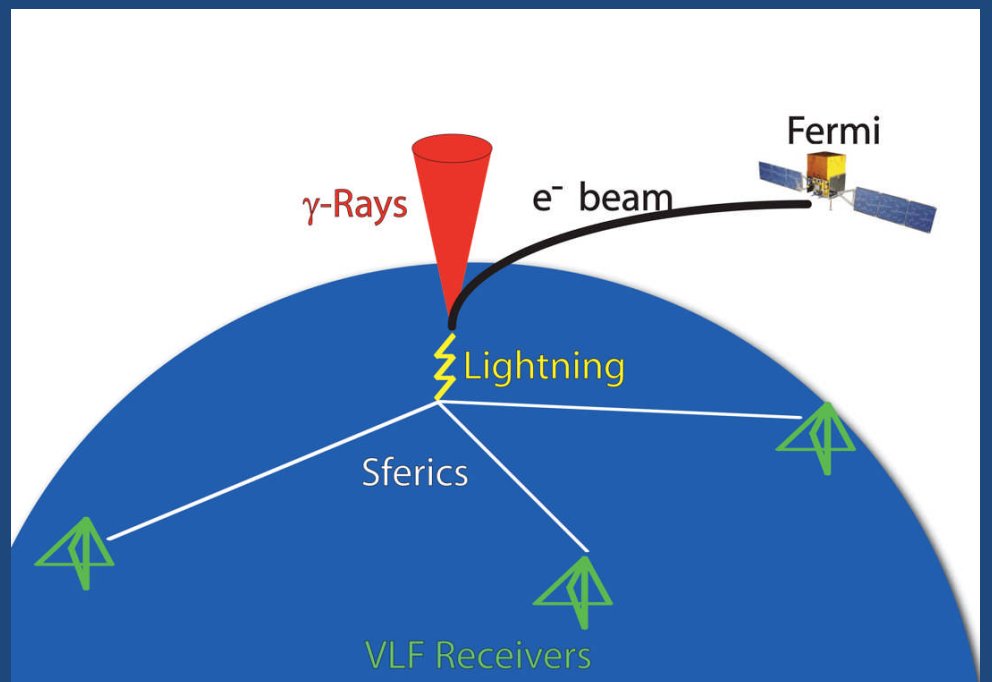




\section{Electron TGFs with GBM}

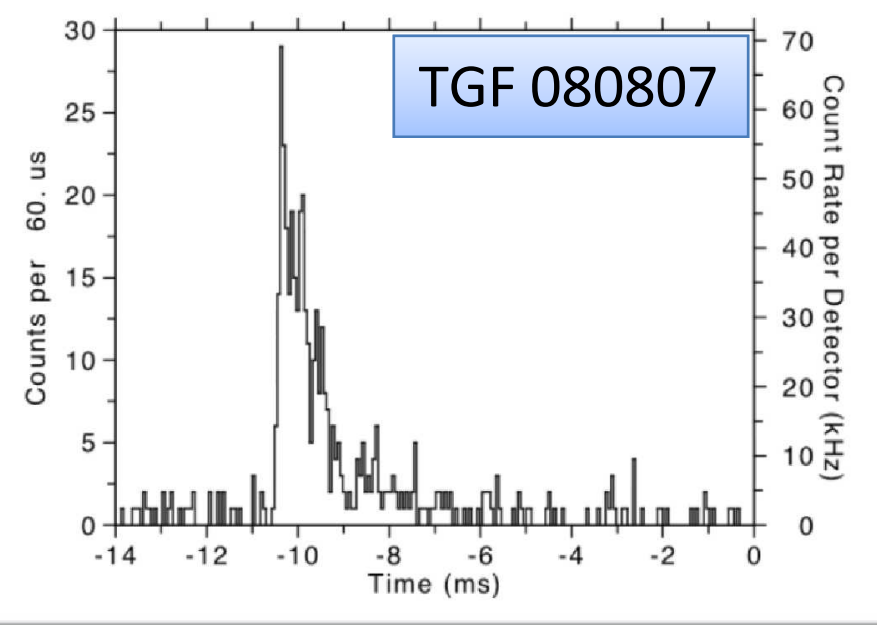

- 4 seen to date with GBM

- Long duration (>1ms)

- Electrons with low pitch angles arrive at spacecraft first

- Low maximum energy $\approx 10 \mathrm{MeV}$

- Lightning activity within $50 \mathrm{~km}$ of magnetic footprint.
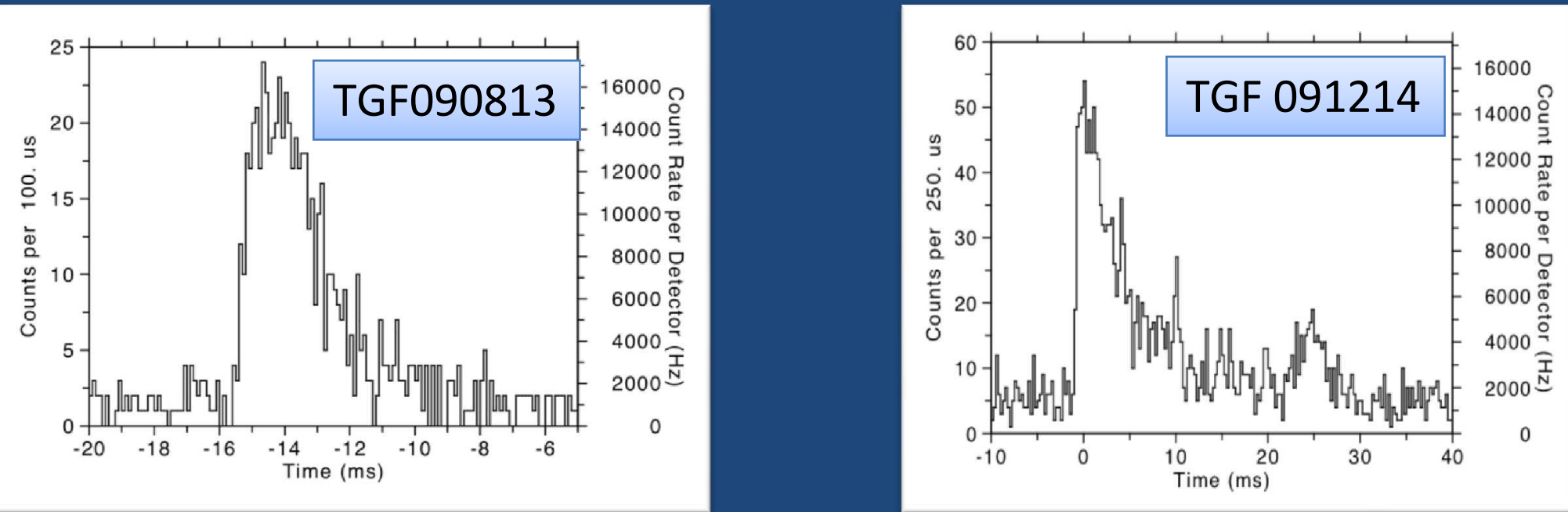


\section{Sferic with Electron TGF!}

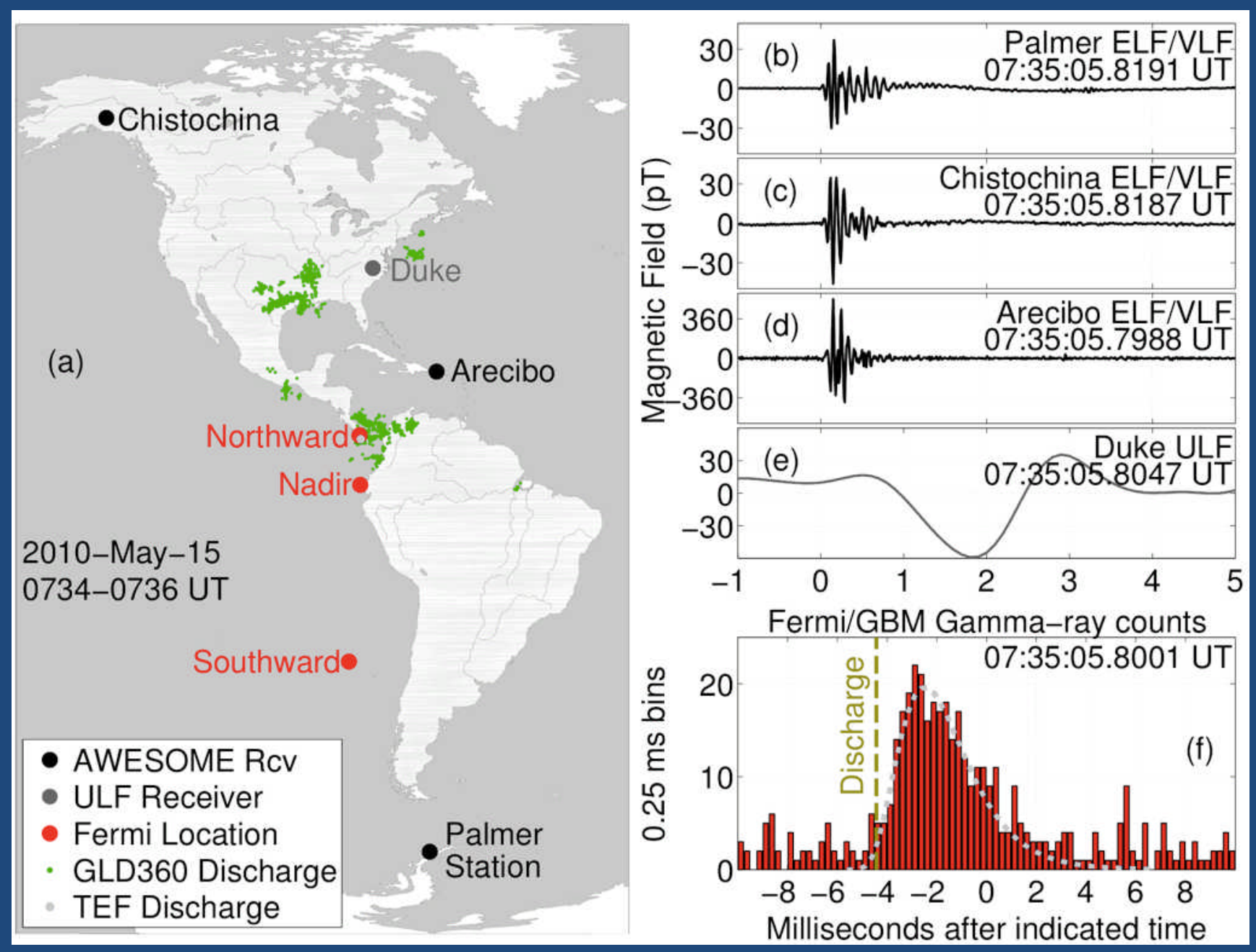

Cohen et al. 2010, to appear in GRL. 


\section{Positron Features Detected with GBM}

TGF 080807

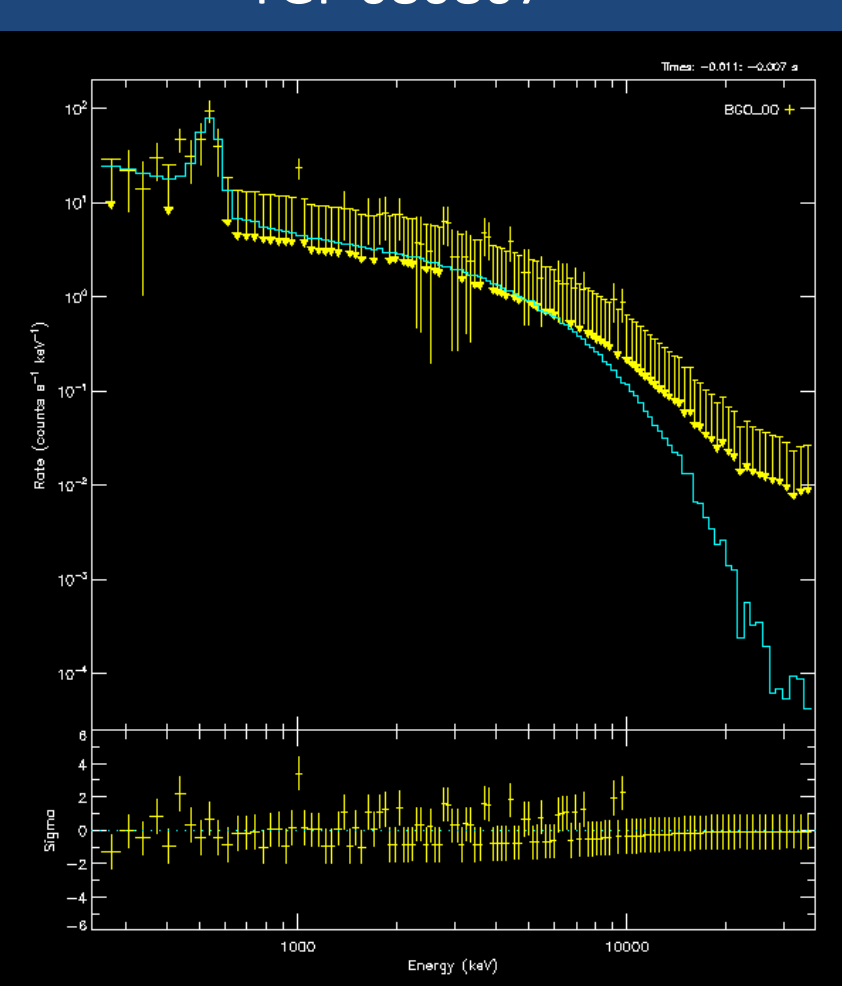

TGF 090813

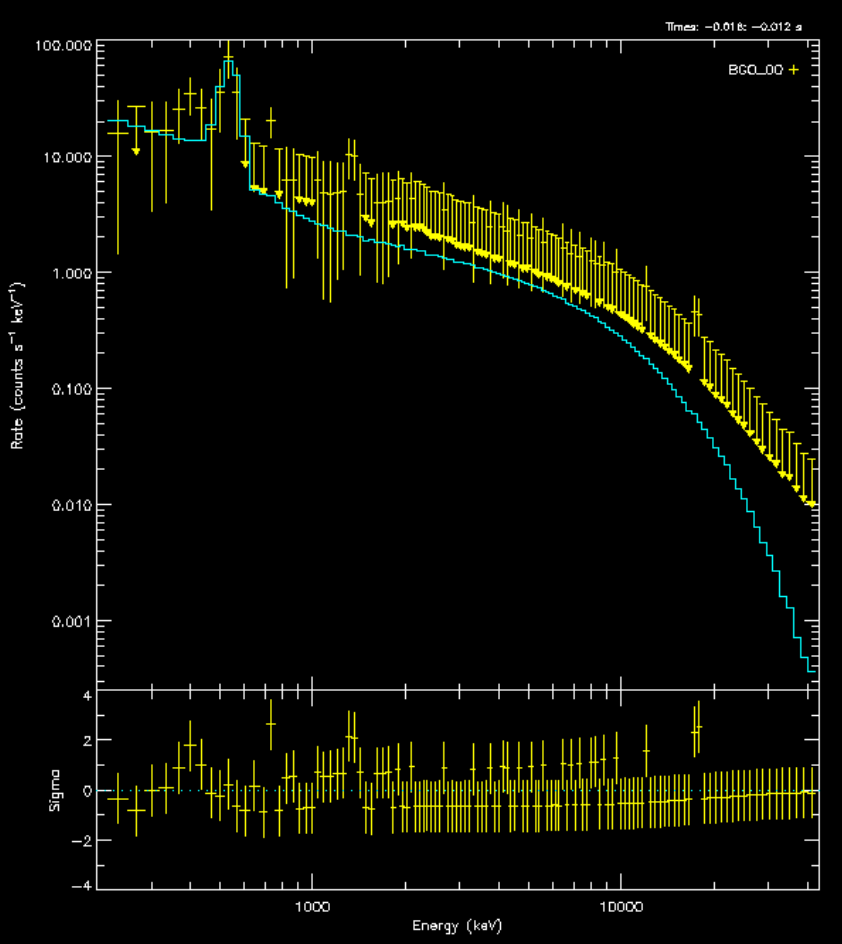

TGF 091214

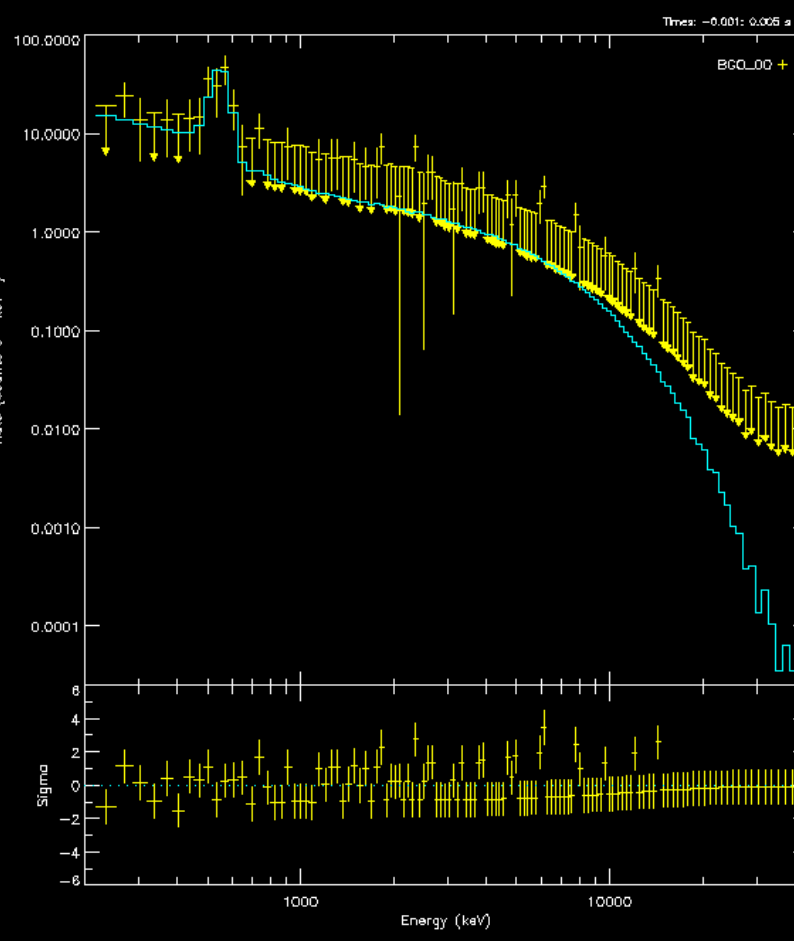

- Spectra fitted by separately simulating electrons and positrons along the field lines.

- Fits require both electron and positron components

- Exponential continuum spectrum with Ecutoff=2-4 MeV. 


\section{Summary}

- TGFs and lightning are simultaneous with no preferred order.

- This supports lightning leader models for TGFs.

- GBM detects gamma-ray TGFs within $300 \mathrm{~km}$ of Fermi's sub-spacecraft location.

- GBM detected electron TGFs within $\sim 50 \mathrm{~km}$ of geomagnetic footprint.

- Positron features detected with GBM from electron TGFs are direct evidence for relativistic phenomena in Terrestrial lightning. 


\section{GBM TGF Papers}

"First Results on Terrestrial Gamma-ray Flashes from the Fermi Gamma-ray Burst Monitor", M. S. Briggs, et al., J. Geophysical Res., in press,

O "Associations between Fermi GBM Terrestrial Gamma-ray Flashes and sferics from the WWLLN", V. Connaughton, et al, to appear in JGR,

"Catalog of Terrestrial Gamma-ray Flashes from the Gamma-ray Burst Monitor on the Fermi Observatory", G. J. Fishman, in preparation,

"Positrons observed from Terrestrial Lightning with Fermi GBM", M. S. Briggs, et al., in preparation, 